\title{
Vol. 68, No. 32
}

In the report "Healthy Contact Lens Behaviors Communicated by Eye Care Providers and Recalled by Patients - United States, 2018," on page 695, the first sentence of the third paragraph should have read "The majority of contact lens-wearing patients surveyed reported wearing soft contact lenses $(\mathbf{8 5 . 8 \%})$. In addition, the majority of patients surveyed were non-Hispanic $(\mathbf{8 5 . 2 \%})$, white $(77.7 \%)$, and female (59.2\%) (Table 1).” 